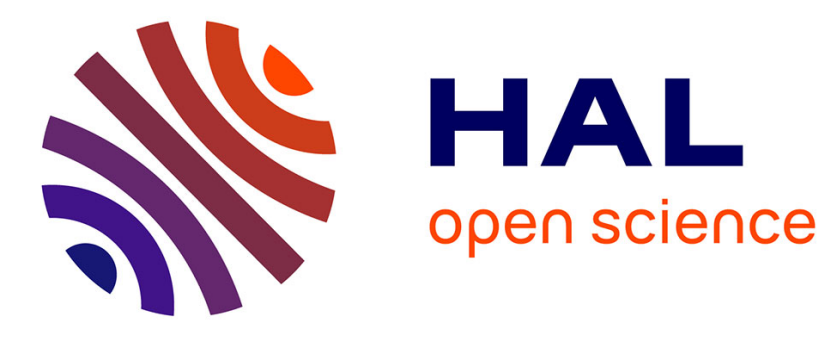

\title{
Numerical simulation of incoherent optical wave propagation in nonlinear fibres
}

\author{
Arnaud Fernandez, Stéphane Balac, Alain Mugnier, Fabrice Mahé, Rozenn \\ Texier-Picard, Thierry Chartier, David Pureur
}

\section{To cite this version:}

Arnaud Fernandez, Stéphane Balac, Alain Mugnier, Fabrice Mahé, Rozenn Texier-Picard, et al.. Numerical simulation of incoherent optical wave propagation in nonlinear fibres. European Physical Journal: Applied Physics, 2013, 64 (2), pp.11. 10.1051/epjap/2013120462 . hal-00797641

\section{HAL Id: hal-00797641 \\ https://hal.science/hal-00797641}

Submitted on 6 Mar 2013

HAL is a multi-disciplinary open access archive for the deposit and dissemination of scientific research documents, whether they are published or not. The documents may come from teaching and research institutions in France or abroad, or from public or private research centers.
L'archive ouverte pluridisciplinaire HAL, est destinée au dépôt et à la diffusion de documents scientifiques de niveau recherche, publiés ou non, émanant des établissements d'enseignement et de recherche français ou étrangers, des laboratoires publics ou privés. 


\title{
Numerical simulation of incoherent optical wave propagation in nonlinear fibres
}

\author{
A. Fernandez ${ }^{1,2}$, S. Balac ${ }^{1,2}$, A. Mugnier ${ }^{3}$, F. Mahé ${ }^{1,4}$, R. Texier-Picard ${ }^{5}$, T. Chartier ${ }^{1,2}$ and D. Pureur ${ }^{3}$ \\ 1 UEB, Université Européenne de Bretagne, Université de Rennes I, 5 Boulevard Laënnec, 35000 Rennes, France \\ 2 CNRS FOTON (UMR 6082), ENSSAT, 6 rue de Kerampont, BP 80518, 22305 Lannion, France \\ 3 QUANTEL, 4 rue Louis de Broglie, 22300 Lannion, France \\ ${ }^{4}$ IRMAR (CNRS UMR 6625), Université de Rennes I, Campus de Beaulieu, 35042 Rennes, France \\ 5 IRMAR (CNRS UMR 6625), ENS Cachan Bretagne, av. Robert Schuman, 35170 Bruz, France
}

\begin{abstract}
Résumé The present work concerns the study of pulsed laser systems containing a fibre amplifier for boosting optical output power. In this paper, this fibre amplification device is included into a MOPFA laser, a master oscillator coupled with fibre amplifier, usually a cladding-pumped high-power amplifier often based on an ytterbium-doped fibre. An experimental study has established that the observed nonlinear effects (such as Kerr effect, four waves mixing, Raman effect) could behave very differently depending on the characteristics of the optical source emitted by the master laser. However, it has not been yet possible to determine from the experimental data if the statistics of the photons is alone responsible for the various nonlinear scenarios observed. Therefore, we have developed a numerical simulation software for solving the generalised nonlinear Schrödinger equation with a stochastic source term in order to validate the hypothesis that the coherence properties of the master laser are mainly liable for the behaviour of the observed nonlinear effects.
\end{abstract}

\section{Introduction}

The MOPFA laser system (Fig. 1) under study aims the generation of very high power $(10 \mathrm{~kW})$ nanosecond pulses at 532 $\mathrm{nm}$ with 50 to $500 \mathrm{kHz}$ repetition rate. This high power pulsed source originates from successive amplification of a master laser source (seeder) emitted at $1064 \mathrm{~nm}$ that will be amplified through a pre-amplification stage and an ytterbium-doped fibre amplifier pumped with a high power laser diode at $976 \mathrm{~nm}$. Once amplification is done, the high power pulses cross the frequency doubling stage to produce nanosecond pulses at 532 $\mathrm{nm}$. However, the frequency doubling stage efficiency can be drastically reduced if the amplified pulses spectrum full width at half maximum (FWHM) exceeds a critical value depending on the physical properties of the doubling stage material. Thus, it is necessary to identify which parameters related to the seeder and/or fibre properties mainly affect the spectrum spreading.

An experimental technique presented in [1], offered us the possibility to measure, throughout temporal slices of the pulse, the spectral evolution of nonlinear effects such as Kerr effect, four waves mixing, Raman effect, for 3 different pulsed fibre laser sources (100 ns of temporal width) generated at $1064 \mathrm{~nm}$ with a repetition rate of $20 \mathrm{kHz}$ and then amplified by an ytterbium doped fiber amplifier. Observation of pulse temporal slices of $10 \mathrm{~ns}$ of duration at different temporal position in the pulse were obtained thanks to an acousto-optical modulator located after the amplifier. By using an optical spectrum analyzer

\footnotetext{
Corresponding author: Stéphane Balac, ENSSAT, 6 rue de Kerampont, CS 80518, 22305 Lannion, France (stephane.balaceuniv-rennes1.fr)
}

it has been possible to characterize the evolution of the nonlinear effects occurring in the fibre amplifier pulse and to discriminate between the nonlinear effects associated with each slice of the pulse.

Whatever was the choice of the source, the initial profile of the laser pulses were quasi-similar (100 ns of temporal width).

The experimental results show that, for the same intensity pulse temporal profile (same temporal width and peak power), there exists very different nonlinear behaviors depending on whether the source is partially coherent with a narrow spectrum or incoherent with a larger spectrum, that is to say from an experimental viewpoint, depending on the characteristics of the master laser oscillator. However these experimental observations do not allow to decide whether the statistics of photons is the only responsible for the various nonlinear scenarios. Hence we have developed a numerical simulation software to study the coherence properties of a fibre amplified pulsed laser source in order to resolve the question.

Wave propagation into an optical fibre with group index $n_{g}$ is governed by the generalised nonlinear Schrödinger equation (GNLSE). This particular form of the Schrödinger equation is obtained from the general set of Maxwell equations taking advantage of a certain number of assumptions made possible from the very specific characteristics of wave propagation in a medium such as a fibre [2]. Among the assumptions that usually can be done for wave propagation into an optical fibre, one is that the optical wave is quasi-monochromatic. It means that the pulse spectrum, centred at pulsation $\omega_{0}$, is assumed to have a spectral width $\delta \omega$ small compared to $\omega_{0}$. Another approximation consists in assuming that the optical wave propagates on 
a single transverse-electric fibre mode and maintains its polarisation along the fibre length so that a scalar model (rather than a full vectorial one) is valid. This is not really the case most of the time but however the approximation works quite well in practice [2]. One of the major assumption, referred to as the slowly varying envelope approximation, concerns the expression of the electric field in the optical fibre. It assumes that the electric field $\mathbf{E}$ is linearly polarised along a direction $\mathbf{e}_{\mathbf{x}}$ transverse to the direction of propagation $\mathbf{e}_{\mathbf{z}}$ defined by the fibre axis and can be represented as a function of time $T$ and position $\mathbf{r}=(x, y, z)$ as

$$
\mathbf{E}(\mathbf{r}, T)=A(z, T) F(x, y) \mathrm{e}^{-\mathrm{i}\left(\omega_{0} T-k z\right)} \mathbf{e}_{\mathbf{x}}
$$

where $A(z, T)$ represents the slowly varying pulse envelope, $F(x, y)$ is the electric wave transverse representation also termed modal distribution and $k$ is the wavenumber. The expression of the modal distribution $F$ can most of the time be computed explicitly using the classical method of separation of variables used for partial differential equations (PDE). For instance, for circular constant transverse section fibres, it can be expressed in terms of Bessel functions [3]. To compute the slowly varying pulse envelope $A$, it is convenient to introduce a frame of reference, called the retarded frame, moving with the pulse at the "group velocity" $v_{g}=c / n_{g}$. The relation between the "local time" $t$ in the retarded frame and the absolute time $T$ is : $t=T-z / v_{g}$. If we introduce as new unknown function $A(z, t)=A\left(z, t+z / v_{g}\right)$ then in the situation considered in this work, the evolution of $A$ is governed by the following form of the GNLSE [2]

$$
\begin{gathered}
\frac{\partial}{\partial z} A(z, t)=-\frac{\alpha(z)}{2} A(z, t)+\left(\sum_{n=2}^{n_{\max }} \mathrm{i}^{n+1} \frac{\beta_{n}}{n !} \frac{\partial^{n}}{\partial t^{n}} A(z, t)\right) \\
+\mathrm{i} \gamma\left[i_{d}+\frac{\mathrm{i}}{\omega_{0}} \frac{\partial}{\partial t}\right] \cdot\left(A ( z , t ) \left(\left(1-f_{R}\right)|A(z, t)|^{2}\right.\right. \\
\left.\left.+f_{R} \int_{0}^{\infty} h_{R}(\tau)|A(z, t-\tau)|^{2} d \tau\right)\right)
\end{gathered}
$$

where $i_{d}$ denotes identity operator. The physical effects for wave propagation in fibre taken into account in (2) are the following. First, some linear effects are expressed through the linear attenuation/gain coefficient $\alpha$ and the linear dispersion coefficients $\beta_{n}, 2 \leq n \leq n_{\max }$ where e.g. $\beta_{2}$ expressed in units $\left[\mathrm{ps}^{2} \mathrm{~km}^{-1}\right]$ accounts for chromatic effects. Non-linear effects are involved in (2) through the nonlinear parameter $\gamma$ expressed in units $\left[\mathrm{W}^{-1} \mathrm{~km}^{-1}\right]$ and defined as $\gamma=\omega_{0} n_{2}\left(\omega_{0}\right) /\left(c A_{\text {eff }}\left(\omega_{0}\right)\right)$ where $n_{2}$ is the nonlinear refractive index for the pulsation $\omega_{0}$ and $A_{\text {eff }}$ known as the effective mode area is defined from the modal distribution $F(x, y)$ as

$$
A_{\mathrm{eff}}=\frac{\left(\iint_{\mathbb{R}^{2}}|F(x, y)|^{2} \mathrm{~d} x \mathrm{~d} y\right)^{2}}{\iint_{\mathbb{R}^{2}}|F(x, y)|^{4} \mathrm{~d} x \mathrm{~d} y} .
$$

In equation (2) first order partial derivation with respect to time takes into account the dispersion of the nonlinearity through the simplified optical shock parameter $\tau_{\text {shock }}=1 / \omega_{0}$. Use of the simplified optical shock parameter is relevant when the guided mode is weakly dependant on the effective area $A_{\text {eff }}$ defined in (3), which is a valid assumption in our study. Instantaneous Kerr effect manifests itself through the term $\left(1-f_{R}\right)|A|^{2}$. The delayed Raman contribution in the time domain is taken into account through the convolution product between the instantaneous power $|A(z, t)|^{2}$ and the Raman time response function for silica-core fibres $h_{R}$. For silica fibres, an expression for $h_{R}$ is proposed in [4]. The constant $f_{R}$ represents the fractional contribution of the delayed Raman response to nonlinear polarisation. It is found to be about 0.2 [5]. As a first step in this study, we haven't taken into account in our model physical phenomena such as amplified spontaneous emission and Raman spontaneous emission.

In order to present our numerical method for solving the GNLSE (2), the following mathematical framework is introduced. We denote by $\mathcal{D}$ the unbounded linear operator

$$
\mathcal{D}: A \mapsto-\frac{1}{2} \alpha A-\sum_{n=2}^{n_{\max }} \beta_{n} \frac{\mathrm{i}^{n-1}}{n !} \partial_{t}^{n} A
$$

and we introduce the nonlinear operator

$$
\mathcal{N}: A \longmapsto \mathrm{i} \gamma \mathcal{T}_{t}\left[\left(1-f_{r}\right) A|A|^{2}+f_{r} A\left(h_{R} \star_{t}|A|^{2}\right)\right]
$$

where $\star_{t}$ stands for the convolution operator with respect to the time variable and $\mathcal{T}_{t}$ refers to the differential operator $\mathrm{i}_{d}+$ $\left(1 / \omega_{0}\right)(\partial / \partial t)$. We are then interested in solving the following PDE problem set over the fibre length $[0, L]$

$$
\left\{\begin{aligned}
\frac{\partial}{\partial z} A(z, t) & =\mathcal{D} A(z, t)+\mathcal{N}(A)(z, t) \quad \forall t \in \mathbb{R} \\
A(0, t) & =a_{0}(t) \quad \forall t \in \mathbb{R}
\end{aligned}\right.
$$

where in the deterministic case the source term $a_{0}$ is assumed to belong to the Hilbert space $\mathbb{L}^{2}(\mathbb{R}, \mathbb{C})$. This PDE problem is not amenable to analytical solution and the use of numerical approximation techniques is required. Classical numerical methods for solving (6) are based on a Split-Step Fourier transform approach and among them the Symmetric Split-Step Fourier method (S3F method) is the most widely used [2], [6], [7]. Recently a "fourth-order Runge-Kutta method in the interaction picture method" (RK4-IP method) has been proposed [8] as a very promising alternative to Split-Step methods for solving the GNLSE. Both methods (S3F and IP) exhibit a computational inner structure very similar and therefore have a computational cost very comparable, although the IP method has an order 4 convergence rate whereas the S3F method is dependent on the second order accuracy of Strang splitting formula and possesses a global quadratic convergence rate [9].

In the present work, the incoherence of the master laser source is modelled by the way of stochastic processes. Namely, the source term $a_{0}$ is assumed to be a complex Gaussian process. As a statistical analysis over a large number of samples is to be achieved once the pulse has propagated along the fibre to determine the characteristics of the pulse amplitude $A$ at the fiber end, we have chosen to implement the RK4-IP method rather than the S3F one to solve the GNLSE in order to decrease the computation time.

The paper is organised as follows. In section 2 we briefly present the Interaction Picture for solving the GNLSE and 


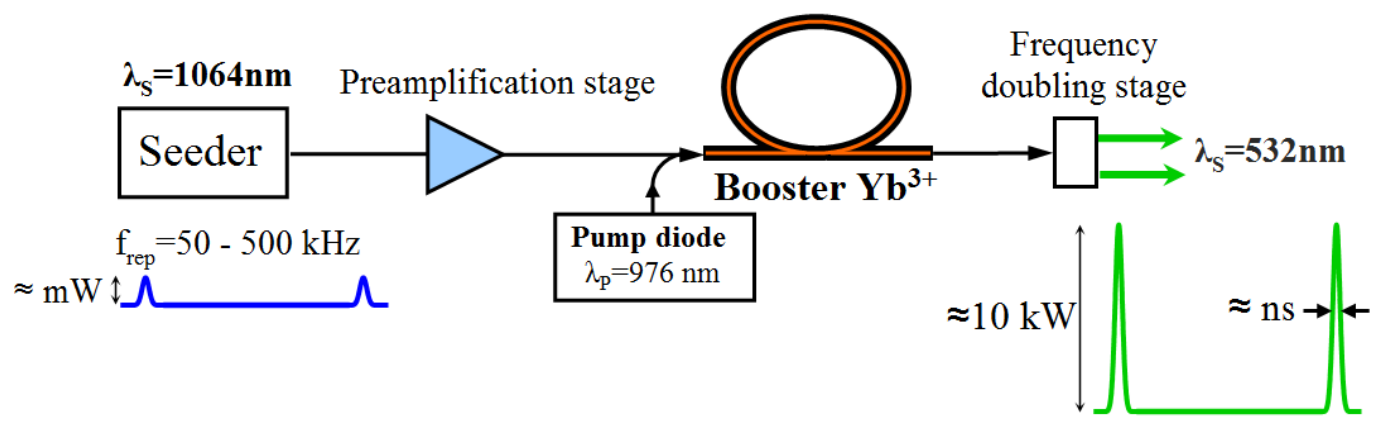

FIG. 1. Schematic of a MOPFA laser with a frequency doubling stage for the generation of high power nanosecond green pulses at few tens of $\mathrm{kHz}$ repetition frequency.

focus on the way the various terms involved in the GNLSE are computed. We present the algorithm of the RK4-IP method used for the purposes of the numerical study of the coherence properties of a fibre laser and we detail the embedded RungeKutta method used for local error estimation and adaptive step size control. In section 3 we present the way the existence of an incoherent optical source is taken into account in the simulation software by introducing complex random processes and we detail the numerical difficulties to overcome. In section 4 we present numerical results obtained with our simulation software and we propose a physical interpretation of the results in the context of the study of coherence properties of a fibre laser.

\section{Solving the GNLSE by RK4-IP method}

Recently a "fourth-order Runge-Kutta method in the interaction picture method" (RK4-IP method) has been presented in [8] together with an experimental comparison of the RK4-IP method to other Split-Step methods based on their numerical efficiency on benchmark problems in optics. The numerical investigation undertaken in [8] indicates that the RK4-IP method exhibits interesting convergence properties and provide more accurate and efficient numerical results than comparable SplitStep methods. In [9] we have investigated the numerical properties of the RK4-IP method from a mathematical point of view; we have exhibited the inner computational structure of the IP method and have compared it to the Symmetric SplitStep method to confirm the numerical comparison results given in [8]. We briefly present in this section the RK4-IP method for solving the GNLSE and we emphasize on the computational setting of the method.

\subsection{Presentation of the RK4-IP method}

\subsubsection{A splitting behind the IP method}

For numerical purposes, the interval $[0, L]$ corresponding to the fibre length is divided into $\mathrm{K}$ sub-intervals where the spatial grid points are denoted by $z_{k}, k=\{0, \ldots, K\}$ such that ] $\left.\left.0, L]=\cup_{k=0}^{K-1}\right] z_{k}, z_{k+1}\right]$ where $0=z_{0}<z_{1}<\cdots<z_{K-1}<$ $z_{K}=L$. The step length between $z_{k}$ and $z_{k+1}$ is denoted $h_{k}$ and we have also set $z_{k+\frac{1}{2}}=z_{k}+\frac{h_{k}}{2}$.
The Interaction Picture method for solving problem (6) consists in solving over each sub-interval $\left[z_{k}, z_{k+1}\right]$ the following 3 nested problems [9]

$$
\left\{\begin{aligned}
\frac{\partial}{\partial z} A_{k}(z, t) & =\mathcal{D} A_{k}(z, t) & \forall z \in\left[z_{k}, z_{k+\frac{1}{2}}\right] \forall t \in \mathbb{R} \\
A_{k}\left(z_{k}, t\right) & =A_{k-1}\left(z_{k}, t\right) & \forall t \in \mathbb{R}
\end{aligned}\right.
$$

where for $k \geq 1$ the mapping $t \in \mathbb{R} \mapsto A_{k-1}\left(z_{k}, t\right)$ represents the solution at grid point $z_{k}$ computed at the previous step $k-1$ and where for $k=0$ we have $A_{-1}\left(z_{0}, t\right)=a_{0}(t) \forall t \in \mathbb{R}$,

$$
\left\{\begin{aligned}
\frac{\partial}{\partial z} A_{k}^{\mathrm{ip}}(z, t) & =\mathcal{G}_{k}\left(z, t, A_{k}^{\mathrm{ip}}\right) & & \forall z \in\left[z_{k}, z_{k+1}\right] \forall t \in \mathbb{R} \\
A_{k}^{\mathrm{ip}}\left(z_{k}, t\right) & =A_{k}\left(z_{k+\frac{1}{2}}, t\right) & & \forall t \in \mathbb{R}
\end{aligned}\right.
$$

where $t \in \mathbb{R} \mapsto A_{k}\left(z_{k+\frac{1}{2}}, t\right)$ represents the solution to problem (7) at point $z_{k+\frac{1}{2}}$ and

$$
\left\{\begin{array}{rlrl}
\frac{\partial}{\partial z} A_{k}(z, t) & =\mathcal{D} A_{k}(z, t) & \forall z & \in\left[z_{k+\frac{1}{2}}, z_{k+1}\right] \forall t \in \mathbb{R} \\
A_{k}\left(z_{k}, t\right) & =A_{k}^{\mathrm{ip}}\left(z_{k+1}, t\right) & \forall t \in \mathbb{R}
\end{array}\right.
$$

where $t \mapsto A_{k}^{\text {ip }}\left(z_{k+1}, t\right)$ represents the solution to problem (8) at grid point $z_{k+1}$. In problem (8), we have set

$$
\mathcal{G}_{k}(z, t, \cdot)=\exp \left(-\left(z-z_{k+\frac{1}{2}}\right) \mathcal{D}\right) \circ \mathcal{N} \circ \exp \left(\left(z-z_{k+\frac{1}{2}}\right) \mathcal{D}\right)
$$

where the exponential terms have to be understood in the sense of the continuous group generated by the unbounded linear operator $\mathcal{D}$.

Solving the sequence of 3 nested problems (7)-(9) over the interval $\left[z_{k}, z_{k+1}\right]$ can be interpreted as doing a change of unknown to solve problem (6) over $\left[z_{k}, z_{k+1}\right]$ taking as new unknown the mapping [9]

$$
A_{k}^{\text {ip }}:(z, t) \in\left[z_{k}, z_{k+1}\right] \times \mathbb{R} \mapsto \exp \left(-\left(z-z_{k+\frac{1}{2}}\right) \mathcal{D}\right) \cdot A_{k}(z, t)
$$

This viewpoint has the advantage of showing why the IP method has better convergence properties than Split-Step based methods. On the contrary to the latter methods where the SplitStep scheme introduces an approximation corresponding to a truncation in the Bakker-Hausdorf formula, with the IP method 
the splitting is exact since it corresponds to a change of unknown.

The major interest in doing such a change of unknown is that on the contrary to problem (6), problem (8) for the unknown $A_{k}^{\text {ip }}$ does not involve explicitly partial derivation with respect to the time variable $t$ and therefore can be numerically solved using standard quadrature schemes for ordinary differential equations such as Runge-Kutta methods. Partial derivation with respect to time now occurs through the operators $\exp \left( \pm\left(z-z_{k+\frac{1}{2}}\right) \mathcal{D}\right)$ that can be computed in a very efficient way by means of Fourier transforms.

We now detail the way the 3 problems (7), (8) and (9) are solved numerically.

\subsubsection{Solving the nonlinear problem (8)}

Problem (8) can be numerically solved by using a standard quadrature scheme for ordinary differential equations such as the fourth-order Runge-Kutta (RK4) scheme defined by the Butcher tableau [10]

$$
\begin{array}{c|cccc}
0 & & & & \\
\frac{1}{2} & \frac{1}{2} & & & \\
\frac{1}{2} & 0 & \frac{1}{2} & & \\
1 & 0 & 0 & 1 \\
\hline & \frac{1}{6} & \frac{1}{3} & \frac{1}{3} & \frac{1}{6}
\end{array}
$$

This Runge-Kutta method is known to have convergence order 4. One step of the RK4 scheme is used to approach the solution to problem (8) at grid point $z_{k+1}$ as follows :

$$
A_{k}^{\mathrm{ip}}\left(z_{k+1}, t\right) \approx A_{k}^{\mathrm{ip}}\left(z_{k}, t\right)+\frac{h_{k}}{6}\left(\alpha_{1}+2 \alpha_{2}+2 \alpha_{3}+\alpha_{4}\right)
$$

where

$$
\begin{aligned}
\alpha_{1} & =\mathcal{G}_{k}\left(z_{k}, t, A_{k}^{\mathrm{ip}}\left(z_{k}, t\right)\right) \\
& =\exp \left(\frac{h_{k}}{2} \mathcal{D}\right) \cdot \mathcal{N}\left(\exp \left(-\frac{h_{k}}{2} \mathcal{D}\right) \cdot A_{k}^{\mathrm{ip}}\left(z_{k}, t\right)\right) \\
\alpha_{2} & =\mathcal{G}_{k}\left(z_{k}+\frac{h_{k}}{2}, t, A_{k}^{\mathrm{ip}}\left(z_{k}, t\right)+\frac{h_{k}}{2} \alpha_{1}\right) \\
& =\mathcal{N}\left(A_{k}^{\mathrm{ip}}\left(z_{k}, t\right)+\frac{h_{k}}{2} \alpha_{1}\right) \\
\alpha_{3} & =\mathcal{G}_{k}\left(z_{k}+\frac{h_{k}}{2}, t, A_{k}^{\mathrm{ip}}\left(z_{k}, t\right)+\frac{h_{k}}{2} \alpha_{2}\right) \\
& =\mathcal{N}\left(A_{k}^{\mathrm{ip}}\left(z_{k}, t\right)+\frac{h_{k}}{2} \alpha_{2}\right) \\
\alpha_{4} & =\mathcal{G}_{k}\left(z_{k}+h_{k}, t, A_{k}^{\mathrm{ip}}\left(z_{k}, t\right)+h_{k} \alpha_{3}\right) \\
& =\exp \left(-\frac{h_{k}}{2} \mathcal{D}\right) \cdot \mathcal{N}\left(\exp \left(\frac{h_{k}}{2} \mathcal{D}\right) \cdot A_{k}^{\mathrm{ip}}\left(z_{k}, t\right)+h_{k} \alpha_{3}\right) .
\end{aligned}
$$

It appears clearly in these formulae that the choice of the point $z_{k+1 / 2}$ in the initial condition of Problem (7) reduces the number of computations of exponential operators, hence the number of Fourier transforms.

\subsubsection{Solving the linear problems (7) and (9)}

The solution to the linear PDE problem (7) in point $z_{k+\frac{1}{2}}$ can formally be written as [9]

$$
\forall t \in \mathbb{R} \quad A_{k}\left(z_{k+\frac{1}{2}}, t\right)=\exp \left(\frac{h_{k}}{2} \mathcal{D}\right) \cdot A_{k}\left(z_{k}, t\right)
$$

where the exponential term has to be understood in the sense of the continuous group generated by the unbounded linear operator $\mathcal{D}$. The mapping $t \in \mathbb{R} \mapsto A_{k}\left(z_{k+\frac{1}{2}}, t\right)$ can be computed very efficiently by means of Fourier transform according to the formula :

$$
\exp \left(\frac{h_{k}}{2} \mathcal{D}\right) \cdot A_{k}\left(z_{k}, t\right)=\mathcal{F}^{-1}\left[\nu \mapsto \widehat{A}_{k}\left(z_{k}, \nu\right) \mathrm{e}^{\widehat{d}_{\nu} \frac{h_{k}}{2}}\right](t)
$$

where $\widehat{A}_{k}\left(z_{k}, \cdot\right)$ denotes the Fourier transform of $A_{k}\left(z_{k}, \cdot\right)$, $\widehat{d_{\nu}}=-\frac{1}{2} \alpha+\mathrm{i} \sum_{n=2}^{n_{\max }} \frac{\beta_{n}}{n !}(2 \pi \nu)^{n}$ and $\mathcal{F}^{-1}$ denotes the inverse Fourier transform operator. The solution to the linear PDE problem (9) at grid point $z_{k+1}$ can also be obtained in a very similar way.

\subsubsection{Computation of the nonlinear terms}

In using the RK4 method for solving problem (8), one needs to compute the nonlinear term (see (5))

$$
\begin{aligned}
\mathcal{N}(A)(z, t) & =\mathrm{i} \gamma\left[\mathrm{id}+\frac{1}{\omega_{0}} \frac{\partial}{\partial t}\right]\left(\left(1-f_{r}\right) A(z, t)|A(z, t)|^{2}\right. \\
& \left.+f_{r} A(z, t)\left(h_{R}(t) \star_{t}|A(z, t)|^{2}\right)\right)
\end{aligned}
$$

This can be achieved in a very efficient way by means of the Fourier transform again since time derivation of functions is then reduced to multiplying the Fourier transform of the function by a factor $-2 \mathrm{i} \pi \nu$. To compute $h_{R}(t) \star_{t}|A(z, t)|^{2}$, we use as well the properties of the Fourier transform with respect to convolution as follows :

$$
h_{R}(t) \star_{t}|A(z, t)|^{2}=\mathcal{F}^{-1}\left[\nu \mapsto \widehat{h}_{R}(\nu) \times|\widehat{A(z, .)}|^{2}(\nu)\right](t) .
$$

Finally, computation of $\mathcal{N}(A)(z, t)$ for all $t \in \mathbb{R}$ and $z \in \mathbb{R}^{+}$ can be achieved through the following steps :

- compute the Fourier transforms $\widehat{h}_{R}$ and $|\widehat{A(z, .)}|^{2}$ of $h_{R}$ and $t \mapsto|A(z, t)|^{2}$ respectively

- multiply these 2 mappings and compute the inverse Fourier transform of the result to obtain the mapping $(z, t) \mapsto h_{R}(t) \star_{t}|A(z, t)|^{2}$

- compute the Fourier transform of the mapping $t \mapsto\left(1-f_{r}\right) A(z, t)|A(z, t)|^{2}+f_{r} A(z, t)\left(h_{R} \star_{t}|A(z, t)|^{2}\right)$

- multiply the result by the mapping $\nu \mapsto \mathrm{i} \gamma\left(1-\frac{2 \mathrm{i} \pi \nu}{\omega_{0}}\right)$

- compute the inverse Fourier transform of this last product.

\subsubsection{Algorithm of the RK4-IP method}

Putting altogether the computational elements given above and after optimizing the whole computational procedure (in particular in order to reduce the number of Fourier transforms to be achieved), we obtain the following algorithm for solving the PDE problem (6) by the RK4-IP method.

\section{RK4-IP algorithm}

Input : Array $u$ contains the sampling of the signal amplitude at step $\mathrm{k}$

Array $\left[\nu_{j}\right]_{j=1, \ldots, J}$ contains the frequency sampling points Array $\left[z_{k}\right]_{k=0, \ldots, K}$ contains the spatial grid points 
Array $\widehat{h}_{R}$ contains the Fourier transform of the Raman response function

\{Initialisation

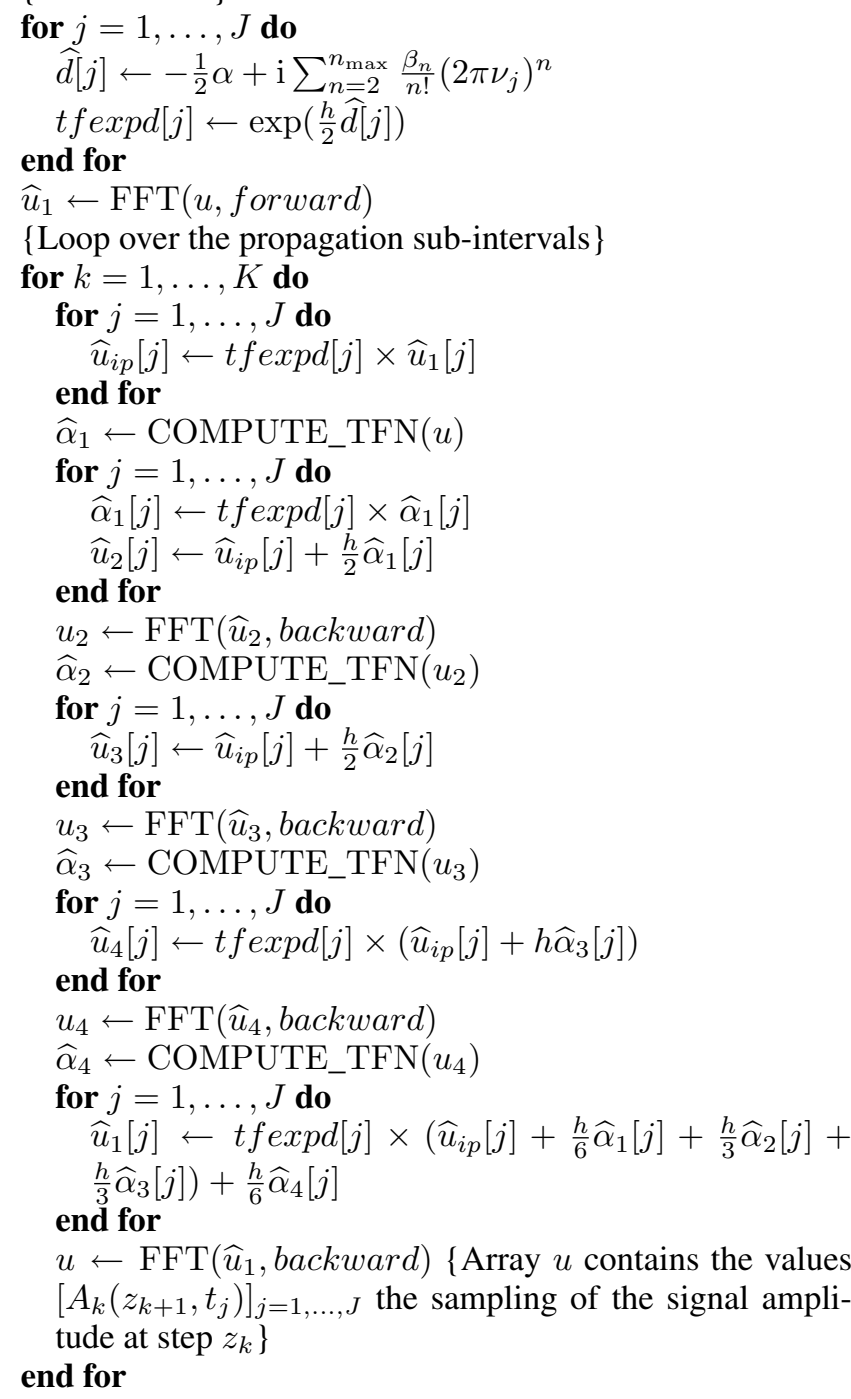

\section{FUNCTION $\widehat{g}=$ COMPUTE_TFN(f)}

$\{$ Compute the Fourier transform of $g: t \mapsto \mathcal{N}(f)(z, t)$ for a given $z$ \}

Input : Array $f$ contains the time sampling of function $f$ for the given $z$

Array $\widehat{h}_{R}$ contains the sampled Fourier transform of the Raman response function

Array $\left[\nu_{j}\right]_{j=1, \ldots, J}$ contains the frequency sampling points

Output : Array $\widehat{g}$ contains the sampled Fourier transform of $g$

for $j=1, \ldots, J$ do

$o p_{1}[j] \leftarrow|f[j]|^{2}$

end for

$\widehat{o} p_{1} \leftarrow \operatorname{FFT}\left(o p_{1}\right.$, forward $)$

for $j=1, \ldots, J$ do

$$
\widehat{o p} p_{2}[j] \leftarrow \widehat{o p} p_{1}[j] \times \widehat{h}_{R}[j]
$$

end for

$o p_{2} \leftarrow \operatorname{FFT}\left(\widehat{o} p_{2}\right.$, backward $)$ \{ Array $o p_{2}$ contains the convolution product $\left.h_{R} \star_{t}|f(t)|^{2}\right\}$

for $j=1, \ldots, J$ do

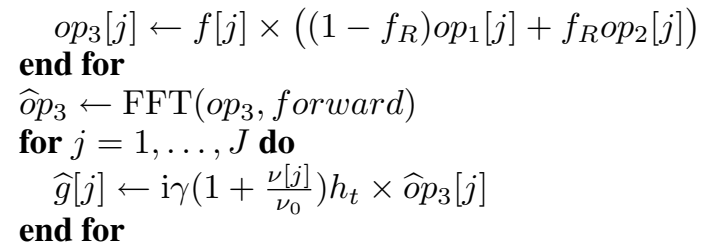

In this algorithm, the computational cost mainly lies in the computation of the Fourier transforms. Over one spatial step, the number of Fourier transforms to be computed is 16 . The C program we have developed to solve the GNLSE (2) by the RK4-IP method according to the above algorithm uses the FFTW library for computing the Fourier transforms [11]. FFTW, for "Fastest Fourier Transform in the West", is a software library for computing discrete Fourier transforms (DFTs) developed by Matteo Frigo and Steven G. Johnson at the Massachusetts Institute of Technology. It supports a variety of algorithms and it can choose the one it estimates or measures to be preferable in the particular circumstances. FFTW is known as the fastest free software implementation of the Fast Fourier transform (FFT) algorithm at present time. It can compute transforms of real-valued and complex-valued arrays of arbitrary size $n$ with a complexity in $\mathcal{O}(n \log (n))$.

\subsection{Embedded Runge-Kutta method for local error estimation}

Any numerical method for solving the GNLSE will perform poorly if the approximate solution is computed on a mesh grid with a constant step $h$. Ideally the step size between 2 successive grid points should be selected automatically to maintain the error lower than a given value in order to achieve both reliability and efficiency of the computations. There are several ways to estimate the local error done in each point of the mesh grid and to select a value for the size of the next step. The most common and general way to estimate the local error is by a process known as "step doubling". To estimate the local error, this method requires for each step the computation of a coarse solution and a fine solution obtained by dividing by 2 the step size used for the coarse solution. This way to estimate the local error is accountable for an over computational cost of around $50 \%$ more than the same method without local error estimate for the same accuracy of the computations. A cheaper adaptive step size method dedicated to the GNLSE is propounded in [12]. It is based on the conservation of a physical quantity termed "the optical photon number" during the propagation of an electromagnetic field along a fibre when linear attenuation and Raman scattering in the fibre are neglected. It is therefore possible to calculate the "photon number error", which is related to the local error, at each step of the computation to retrieve information about the numerical error over one computational step of the IP method applied to GNLSE. The interest of this approach is that the computation of the photon number error can be done at a very cheap numerical cost. However strictly speaking this method is only valid for lossless fibers or for linear losses fibers.

When using Runge-Kutta methods an alternative way to compute the local error, well documented in the literature on 
RK methods where it is referred as the "embedded RungeKutta methods" exists, see e.g. [10]. This way of estimating the local error, inherent to the RK methods themselves, do not require any assumption on the model and is not liable for extra computational cost. It is this method we have chosen to implement in our simulation software. A detailed theoretical and experimental comparison of the three above mentioned approaches for local error estimation has been achieved and is presented in [13].

\subsubsection{Principle of Embedded Runge-Kutta methods}

In this work we have chosen to estimate the local error by using an embedded Runge-Kutta method [10]. Embedded Runge-Kutta (ERK) methods are special Runge-Kutta (RK) methods designed to deliver 2 approximations of the solution of the initial value problem under consideration corresponding to 2 RK schemes of different convergence orders. These 2 approximations of the solution can be considered as an accurate approximate solution (the one computed with the numerical scheme of higher order) and a coarse approximate solution (the one computed with the one of lower order). For efficiency, the 2 RK schemes bear several computational stages in common in order to reduce the computational cost of the local error estimation. Here we have considered the ERK method given in one hand by the fourth order RK scheme (RK4) defined by Butcher tableau (10) and on the other hand by the third order RK scheme (RK3) defined by the following Butcher tableau

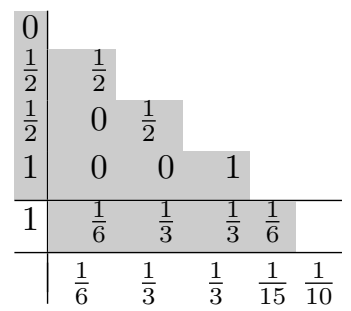

where the cells highlighted in gray are shared with Butcher tableau (10) for the standard RK4 method. This embedded RK4(3) method actually coincides with Dormand and Prince RungeKutta 4(3) $T$ method [14].

Assuming that the solution value at grid point $z_{k}$ is regarded as exact (because we are concerned by an estimation of the local error), we denote by $A_{k+1}^{[3]}$ (resp. $A_{k+1}^{[4]}$ ) the approximate solution computed at the current grid point $z_{k+1}$ by the third order (resp. the fourth order) Runge-Kutta method. The local errors for each of the 2 methods are respectively given by

$$
\begin{aligned}
& A\left(z_{k+1}, t\right)-A_{k+1}^{[3]}(t)=\psi_{3}\left(t, z_{k}, A_{k}^{[3]}\right) h_{k}^{4}+\mathcal{O}\left(h_{k}^{5}\right) \\
& A\left(z_{k+1}, t\right)-A_{k+1}^{[4]}(t)=\psi_{4}\left(t, z_{k}, A_{k}^{[4]}\right) h_{k}^{5}+\mathcal{O}\left(h_{k}^{6}\right)
\end{aligned}
$$

where $\psi_{3}$ (resp. $\psi_{4}$ ) is a function of the elementary differentials of order 4 (resp. 5) [10] of the function $\mathcal{G}_{k}$ involved in problem (7). Therefore by difference of these 2 relations we obtain

$$
A_{k+1}^{[4]}(t)-A_{k+1}^{[3]}(t)=\psi_{3}\left(t, z_{k}, A_{k}^{[3]}\right) h_{k}^{4}+\mathcal{O}\left(h_{k}^{5}\right) .
$$

Thus from (12) and (13) the local error for the RK3 method at grid point $z_{k+1}$ can be approximated with an error in $\mathcal{O}\left(h_{k}^{5}\right)$ in the following way

$$
\ell_{k+1}(t) \approx A_{k+1}^{[4]}(t)-A_{k+1}^{[3]}(t) .
$$

The advantages of estimating the local error through the ERK4(3) method defined by Butcher tableau (11) compared to other embedded RK schemes are the following. The ERK4(3) method preserves all the nice features of the RK4 scheme when used with the IP method as described in paragraph 2.1.2. Moreover the computation of both terms $A_{k+1}^{[4]}$ and $A_{k+1}^{[3]}$ in the ERK4(3)-IP method can be achieved at no extra cost compare to the RK4-IP method without local error estimation (namely, the extra cost for each step is negligible since it is limited to 2 additions and 3 multiplications and the need to keep in memory 2 intermediate results). The implementation of the ERK4(3)IP method requires little modification of the RK4-IP algorithm presented in paragraph 2.1.5 and we refer to [15] for a comprehensive presentation of the ERK4(3)-IP algorithm.

Finally, the $\mathbb{L}^{2}$-local error at grid point $z_{k+1}$ is computed as follows

$$
\begin{aligned}
\left\|\ell_{k+1}\right\|_{\mathbb{L}^{2}} & \approx\left(\int_{\mathbb{R}}\left|A_{k+1}^{[4]}(t)-A_{k+1}^{[3]}(t)\right|^{2} \mathrm{~d} t\right)^{\frac{1}{2}} \\
& \approx\left(h_{t} \sum_{j=0}^{J-1}\left|A_{k+1}^{[4]}\left(t_{j}\right)-A_{k+1}^{[3]}\left(t_{j}\right)\right|^{2}\right)^{\frac{1}{2}}
\end{aligned}
$$

where $\left(t_{j}\right)_{j=0, \ldots, J}$ denotes a constant step size sampling $h_{t}$ of the observed time period and the last approximation results from the use of the left rectangle quadrature rule. We want to mention that even though the local error estimation (14) strictly holds only for the third order method, in practice we use the value given by the fourth order method as the approximation of the solution at grid point $z_{k}$. In general, it slightly overestimates the actual local error, which is safe but not fully optimal.

\subsubsection{Step-size control}

For step-size control, a tolerance is given as a bound for the norm of the local error estimation. One step-size control strategy [10] consists in rejecting the current step-size if it gives an estimated local error higher than the specified tolerance and in accepting the solution computed with this step-size otherwise. When the current step-size is rejected, a new smaller step-size has to be chosen to recompute the solution over the current step. Moreover when the current step-size meets the tolerance requirement for the local error it has to be scaled up for the next step computations. In both cases, the new step-size has to be estimated using the available information on the previous step computations.

For robustness purposes, the step-size control has to be designed in order to respond as smoothly as possible with real or apparent abrupt changes in behaviour. This means that the stepsize should not vary from one step to the other by an excessive ratio. In order to avoid situations where the specified tolerance is ever exceeded resulting in rejecting too many steps, a safety 
factor is introduced. If $h$ is the value of the step-size estimated to give a predicted truncation error equal to the tolerance, then the smaller value $0.9 \mathrm{~h}$ is used instead. Following these requirements, the step-size control formula proposed in [10] reads

$$
h_{\text {new }}=\max \left(0.5, \min \left(2.0,0.9\left(\frac{\text { tol }}{\text { est }}\right)^{\frac{1}{3}}\right)\right) h_{\text {old }} \text {. }
$$

where "tol" denotes the tolerance value specified by the user as a bound of the local error and "est" denotes the estimation of the local error for the current step computed from (15). The 3 constants with values $0.5,2.0$ and 0.9 are somewhat arbitrary and have to be regarded as design parameters.

\section{Simulation of incoherent laser sources by random process}

In this work we have chosen to model the incoherence behaviour of the optical source by using random processes following in this a seminal work of B. Gross and J. T. Manassah [16]. Thus the electric field amplitude $A$ is now considered as a complex random process $A_{z, t}$ indexed by the space variable $z \in[0, L]$ and by the time variable $t \in \mathbb{R}$.

\subsection{Complex random processes}

The electric field amplitude $A$ is a complex random process indexed by the set $[0, L] \times \mathbb{R}$ over a probability space $(\Omega, \mathcal{F}, \mathbb{P})$; that is to say $A$ is now considered as a mapping from the sample space $\Omega$ into a set of functions from $[0, L] \times \mathbb{R}$ into $\mathbb{C}$,

$$
A: \varpi \in \Omega \mapsto\left(A_{\varpi}:(z, t) \in[0, L] \times \mathbb{R} \mapsto A(z, t, \varpi) \in \mathbb{C}\right) .
$$

The value of $A$ at time $t$ and position $z$ for one observed "experiment" is a complex number. When the experiment is repeated, this value is assumed to vary randomly according to the marginal probability distribution of the process. The random process $A$ involves two types of variables, one "probabilistic" variable corresponding to an outcome $\varpi$ in the sample space $\Omega$ and one "deterministic" variable $(z, t) \in[0, L] \times \mathbb{R}$ and

- for every fixed outcome $\varpi_{0} \in \Omega$, the function $A_{\varpi_{0}}:(z, t) \in[0, L] \times \mathbb{R} \mapsto A\left(z, t, \varpi_{0}\right) \in \mathbb{C}$ is a "deterministic" function in the usual sense ;

- for every fixed value $\left(z_{0}, t_{0}\right) \in[0, L] \times \mathbb{R}$ the function $A_{z_{0}, t_{0}}: \varpi \in \Omega \mapsto A\left(z_{0}, t_{0}, \varpi\right) \in \mathbb{C}$ is a random variable Moreover, for a fixed value $z_{n} \in[0, L]$, the mapping

$$
A^{\left[z_{n}\right]}: \varpi \in \Omega \mapsto\left(A_{\varpi}^{\left[z_{n}\right]}: t \in \mathbb{R} \mapsto A\left(z_{n}, t, \varpi\right) \in \mathbb{C}\right)
$$

is itself a complex random process.

The mean function of the complex random process $A^{\left[z_{n}\right]}$ is defined as

$$
m_{A^{\left[z_{n}\right]}}: t \in \mathbb{R} \mapsto \mathbb{E}\left(A_{z_{n}, t}\right) \in \mathbb{C}
$$

where $\mathbb{E}$ denotes the expectation function. When it has a sense, the variance of the complex random process $A^{\left[z_{n}\right]}$ is defined by

$$
\operatorname{Var}_{A\left[z_{n}\right]}: t \in \mathbb{R} \mapsto \mathbb{E}\left(\left|A_{z_{n}, t}-\mathbb{E}\left(A_{z_{n}, t}\right)\right|^{2}\right) \in \mathbb{R}^{+}
$$

The covariance function of $A^{\left[z_{n}\right]}$ is the mapping

$$
\begin{aligned}
\operatorname{Cov}_{A^{\left[z_{n}\right]}} & :\left(t_{1}, t_{2}\right) \in \mathbb{R}^{2} \mapsto \\
& \mathbb{E}\left(\left(A_{z_{n}, t_{1}}-\mathbb{E}\left(A_{z_{n}, t_{1}}\right)\right) \overline{\left(A_{z_{n}, t_{2}}-\mathbb{E}\left(A_{z_{n}, t_{2}}\right)\right)}\right) .
\end{aligned}
$$

In this study we have assumed for all $z_{n} \in[0, L]$ the complex random process $A^{\left[z_{n}\right]}$ to be weak-sense (or wide-sense) stationary that is to say its mean function is identically constant and its covariance function is translation invariant, i.e. $\forall \tau \in \mathbb{R}$, $\forall\left(t_{1}, t_{2}\right) \in \mathbb{R}^{2}$

$$
\operatorname{Cov}_{A^{\left[z_{n}\right]}}\left(t_{1}+\tau, t_{2}+\tau\right)=\operatorname{Cov}_{A^{\left[z_{n}\right]}}\left(t_{1}, t_{2}\right) .
$$

For such random processes the covariance function actually only depends on the gap between the 2 variables rather than on the values of these variables themselves and it is usual to call covariance function of the process the mapping $C_{A^{\left[z_{n}\right]}}: t \in \mathbb{R} \mapsto \operatorname{Cov}_{A^{\left[z_{n}\right]}}(t, 0)$ instead of the function $\operatorname{Cov}_{A^{\left[z_{n}\right]}}$ itself. The covariance function $C_{A^{\left[z_{n}\right]}}$ is positive definite.

\subsection{Distribution of the source random process}

The source field amplitude $A_{0, t}$ is assumed to be a zero mean stationary Gaussian complex random process defined by its covariance function $\Gamma=C_{A^{\left[z_{0}\right]}}$ that is to say we assume $\forall n \in \mathbb{N}^{*}, \forall\left(t_{1}, \ldots, t_{n}\right) \in \mathbb{R}^{n}$ the $\mathrm{n}$ dimensional complex random vector $\left(A_{0, t_{1}}, \ldots, A_{0, t_{n}}\right)$ has a zero mean multivariate Gaussian distribution $\mathcal{N}(0, \Sigma)$ where the covariance matrix $\Sigma$ has entries elements $\Sigma_{i j}=\Gamma\left(t_{i}-t_{j}\right)$.

\subsubsection{Basic ideas of the simulation approach}

From a mathematical point of view it is impossible to predict the law of the random process $A_{z, t}$ at any position $z$ in the fibre due to the nonlinearities in the propagation equation (2). We therefore have to restrict ourselves to the computation of the mean and correlation functions of the random process $A_{L, t}$ at the fibre end.

The basic idea of the simulation approach is to randomly draw an outcome $A_{0, t}(\varpi)$ for the source electric field amplitude and to use the propagation equation (2) to compute the electric field amplitude $A_{z, t}(\varpi)$ at any position $z$ along the optical fibre and at any time $t$. If a large number of outcomes are considered simultaneously, then by statistical averaging it is possible to compute an approximation of the mean and correlation functions of the random process $A_{L, t}$.

Once the time interval has been sampled with a constant time step $h_{t}$ and discrete times $t_{j}=j h_{t}, j \in\{0, \ldots, J\}$ have been introduced, the continuous zero mean circular stationary Gaussian complex random process $A_{0, t}$ is handled through a zero mean Gaussian complex random vector $\mathbf{X}=\left(X_{t_{0}}, \ldots, X_{t_{J}}\right)$ where the random variable $X_{t_{j}}$ is defined by $X_{t_{j}}=A_{0, t_{j}}$. The elements of the covariance matrix $\Sigma$ of the random vector $\mathbf{X}$ are given by $\Sigma_{i j}=\Gamma\left(t_{j}-t_{i}\right)$ and the covariance matrix is hermitian positive definite and Toeplitz. To simulate a multivariate Gaussian distribution $\mathcal{N}(0, \Sigma)$ one can proceed as follows. Let 
$\mathbf{Y}=\left(Y_{0}, \ldots, Y_{J}\right)$ be a random vector where the vector components $Y_{j}, j \in\{0, \ldots, J\}$ are independent normal random variables $\mathcal{N}(0,1)$ and let $\mathrm{L} \in \mathcal{M}_{J+1}(\mathbb{C})$ be the unique nonsingular lower triangular matrix with positive diagonal entries such that $\Sigma=\mathrm{L} \mathrm{L}^{\mathrm{H}}$ (such a Cholesky factorisation exists since the covariance $\Sigma$ is hermitian positive definite and the matrix $\mathrm{L}$ is referred to as the square root of $\Sigma$ and denoted $\Sigma^{\frac{1}{2}}$ ). Then the random vector $X=\mathrm{L} Y$ has a multivariate Gaussian distribution with covariance matrix $\Sigma$ and zero mean. Due to the intrinsic dynamic of the propagation equation (2) in the experimental situation considered here, it is necessary to have a time discretisation (or correspondingly a frequency one) with a large number of grid points. Indeed, the pulse-width of the studied laser is closed to a nanosecond with a spectral width covering few $\mathrm{THz}$ at fibre output due to strong occurrence of nonlinearities (self-phase modulation, Raman scattering) through propagation. Hence, for a spectral resolution allowing a spectral accuracy close to the laser longitudinal modes spacing $(\approx 10$ $\mathrm{MHz}$ ), the number of grid points should be as high as $2^{23}$.

\subsubsection{Generation of the source Gaussian complex random vector}

In their work on modelling the propagation of incoherent laser sources in nonlinear fibres [16], Gross and Manassah used the "factorisation method" for generating Gaussian complex random vectors based on the Cholesky decomposition of the covariance matrix presented above. The main drawback of this numerical method is that the Cholesky decomposition of the covariance matrix $\Sigma$ may fail for numerical reasons. Indeed although the covariance matrix $\Sigma$ is hermitian positive definite, and the Cholesky decomposition exists, in practise for covariance matrices of large size, their smallest eigenvalues decay very rapidly toward 0 and the covariance matrix is almost singular. From a numerical point of view, the covariance matrix $\Sigma$ is therefore hermitian positive but not definite and the numerical implementation of the Cholesky method fails to compute the Cholesky decomposition of the covariance matrix $\Sigma$. This kind of behaviour is observed for instance for Gaussian correlation functions $\Gamma(t)=a \mathrm{e}^{-b t^{2}}$. Moreover this method requires $\mathcal{O}\left(J^{2}\right)$ numerical operations to generate one outcome of a Gaussian vector of size $\mathrm{J}$ and hence it is computationally expensive for large values of J. In the 1990's alternative numerical methods to the "factorisation method" have been investigated for the simulation of large samples according to a multivariate Gaussian distribution. A method of choice is the "Circular embedding method" (CEM) independently invented by Dietrich and Newsam [17] and Wood and Chan [18]. The CEM is the fastest algorithm for unconditional simulation and it is "exact in principle" which means that it is exact under the assumptions that no error in the computer arithmetic occurs and that truly independent random variables are used.

The idea behind the CEM for simulating a zero mean multivariate Gaussian random vector is the following. The covariance matrix $\Sigma$ which has the property of being a Toeplitz matrix is embedded in a hermitian circulant matrix $\mathrm{C} \in \mathcal{M}_{K}(\mathbb{C})$. Calculation of the square root $\mathrm{C}^{\frac{1}{2}}$ of a circulant nonnegative definite matrix may be performed efficiently using the Fast Fourier Transform [17][18]. Moreover if $\mathbf{Y}=\left(Y_{1}, \ldots, Y_{K}\right)$ is a random vector where the components $Y_{k}, k \in\{1, \ldots, K\}$ are independent normal random variables $\mathcal{N}(0,1)$, then the circulant matrix can be constructed in such a way that a selected sub-vector $X \in \mathbb{C}^{J+1}$ of $Z=\mathrm{C}^{\frac{1}{2}} Y \in \mathbb{C}^{K}$ has a multivariate Gaussian distribution with covariance matrix $\Sigma$ and zero mean [17][18]. The algorithm of the CEM can be found in [19]. In this work we use the adaptation of the CEM for complex Gaussian random vector [20].

\subsection{Statistical analysis of the final complex random vector}

As mentioned before, the distribution law of the random process $A_{L, t}$ at the fibre end cannot be predicted by the theory from the distribution law of the source random process $A_{0, t}$. We therefore restrict ourselves to statistical estimation of its mean and correlation functions from a large number $\mathrm{M}$ of outcomes $A_{L, t}(\varpi)$. As the mean of a stationary random process is time invariant, we may estimate the mean of $A_{L, t}$ by the cumulative formula

$$
m_{L} \approx \frac{1}{M} \frac{1}{J+1} \sum_{j=0}^{J} \sum_{m=1}^{M} A_{L, t_{j}}\left(\varpi_{m}\right) .
$$

The covariance matrix $\Sigma_{L}$ of the complex random vector $\left(A_{L, t_{j}}\right)_{j=0 \cdots, J}$ is hermitian and has upper triangular entries $(j \geq i)$ given by

$$
\begin{aligned}
\Sigma_{i j} & =\mathbb{E}\left(A_{L, t_{i}} \overline{A_{L, t_{j}}}\right)-m_{L}^{2}=\Gamma\left(t_{j}-t_{i}\right)-m_{L}^{2} \\
& =\Gamma\left((j-i) h_{t}\right)-m_{L}^{2}
\end{aligned}
$$

where for all $k \in\{0, \ldots, J\}$

$$
\Gamma\left(k h_{t}\right) \approx \frac{1}{M} \frac{1}{J-k} \sum_{j=0}^{J-k} \sum_{m=1}^{M} A_{L, t_{j}}\left(\varpi_{m}\right) \bar{A}_{L, t_{j+k}}\left(\varpi_{m}\right) .
$$

\section{Numerical results}

The numerical simulation results presented in this section have been not only obtained in a record time through the choice of an efficient and fast method (RK4-IP) but also thanks to the kind help of Project team CORDIAL from IRISA (CNRS UMR 6074), it has been possible to run our simulations on a multi-core processing unit with 64 physically separated processors and 132 Go RAM. Hence it has been likely to optimize computation time by performing multi-threaded FFT, a routine for shared-memory parallel hardware handled by FFTW library [11]. This option was useful to save computation time since RK4-IP computations involved a time/frequency number of sampling points exceeding $2^{19}$. Moreover, as explained in section 3.3, generation and propagation of a large number of outcomes $(\approx 2000)$ of the random process is required. Regarding this, an additive gain of time is possible through independent generation and simultaneous propagation of multiple outcomes of the random process, which appears as an alternative computation technique. Its features help to drastically 
reduce computing time. For instance, with a number of $2^{19}$ time sampling points the computing time for the generation and propagation of 2000 outcomes takes roughly 1 hour and a half through the parallel computation of 50 parallel outcomes, including an adaptive step-size control strategy with tolerance tol $=10^{-7}$ but without FFT multi-threading.

Some valuable physical statements can be deduced from numerical simulation experiments. Namely, important informations regarding the impact of fibre linearities and nonlinearities on partially coherent (PC) laser source can be obtained. As well, the design of a PC laser source is made convenient since some of its physical features such as spectrum width and shape can be adjusted through the setting of the correlation function width and shape.

The simulation parameters used in this paper are related to the propagation and amplification of high power (hundreds of Watts) PC super Gaussian pulse with $T_{p}=0.5$ ns half-width into $3 \mathrm{~m}$ of $\mathrm{Yb}^{3+}$ doped active fibre (booster) exhibiting an amplification gain ranging from 10 to $20 \mathrm{~dB}$ depending on the laser diode pump power. As mentioned in the introduction, we want to investigate the impact of the PC source coherence time $t_{\text {coh }}$ on its amplification.

Results depicted in Fig. 2 show the outgoing mean power spectrum density (PSD) [21] after $3 \mathrm{~m}$ of propagation and 18.6 $\mathrm{dB}$ amplification $\left(P_{\text {pump }}=35 \mathrm{~W}\right)$ in the booster for different values of the booster Kerr parameter $\gamma$ ranging from 0.2 to 0.8 $\mathrm{W}^{-1} \mathrm{~km}^{-1}$. The input mean PSD shape is Lorentzian and it corresponds to a complex Gaussian random process generated at a carrier frequency $\lambda_{S}=1064 \mathrm{~nm}$ with an exponential covariance function and a coherence time $t_{\mathrm{coh}}=2.65 \mathrm{ps}$. As the Kerr effect is increasing, PSD spreading and Raman shift tend to grow bigger.

The input field's covariance function is given by

$$
C_{A^{\left[z_{n}\right]}}(\tau)=\exp \left(-\left|\tau / t_{\text {coh }}\right|\right)
$$

By virtue of the Wiener-Khinchin theorem in the very special case of a wide-sense stationary complex random process, the covariance function and the PSD form a Fourier transform pair [21]. Hence, the input PSD (IN) is Lorentzian with a full width at half maximum (FWHM) $\Delta \nu_{\text {IN }}$ depending on $t_{\text {coh }}$ through the relationship :

$$
\Delta \nu_{\mathrm{IN}}=\frac{1}{\pi t_{\mathrm{coh}}}
$$

With an initial FWHM $\Delta \nu_{\mathrm{IN}}=120 \mathrm{GHz}$, we can appreciate in Fig. 2 a noticeable behavior through propagation in active fibre. Our software allows not only to appreciate and quantify the PSD broadening around $\lambda_{S}$ due to self-phase modulation (SPM), but also it allows the quantification of the Raman induced frequency shift (RIFS) which is made possible with the choice of a wide spectral window (200 nm) [2]. The RIFS is an inelastic phenomenon which appears as a transfer of energy from high frequency components $\lambda_{S}$ of a pulse to the lowerfrequency components of the same pulse. Regarding our experimental setup (Fig. 1), Raman shift and SPM represent deleterious effects leading to spectrum broadening responsible for a decreasing efficiency at the frequency doubling stage. Thus, booster amplifiers and PC laser properties lead to more or less emphasize those nonlinearities. Regarding the Raman energy quantity, comparison with previous work [1] shows a lower quantity of energy. We suspect that numerical computation of Raman spontaneous energy added to amplified spontaneous emission can drastically increase the amount of spectral energy above $1100 \mathrm{~nm}$.

A closer insight showed in Fig. 3 completes the results presented in Fig. 2 and certifies that the phenomenon of spectrum spreading is responsible for a poorer energy confinement as $\gamma$ is increasing which is detrimental to an optimum frequency doubling. We can notify that energy confinement decreases proportionally with $\gamma$. Indeed, half of the output signal energy is confined into a $1 \mathrm{~nm}$ spectral window for $\gamma=0.2 \mathrm{~W}^{-1} \mathrm{~km}^{-1}$, this same energy is confined into a $4 \mathrm{~nm}$ spectral window when $\gamma$ is four times bigger.

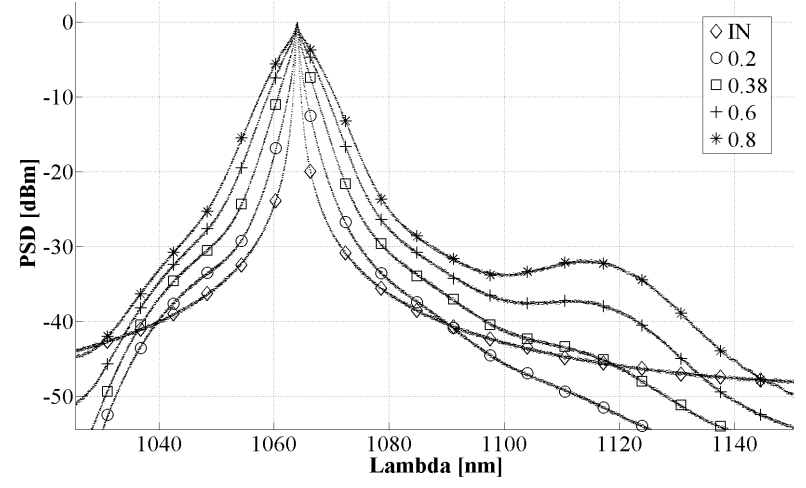

FIG. 2. Fibre amplified PSD according to the fibre Kerr parameter $\gamma$ ranging from 0.2 to $0.8 \mathrm{~W}^{-1} \mathrm{~km}^{-1}$. IN stands for the Lorentzian input PSD.

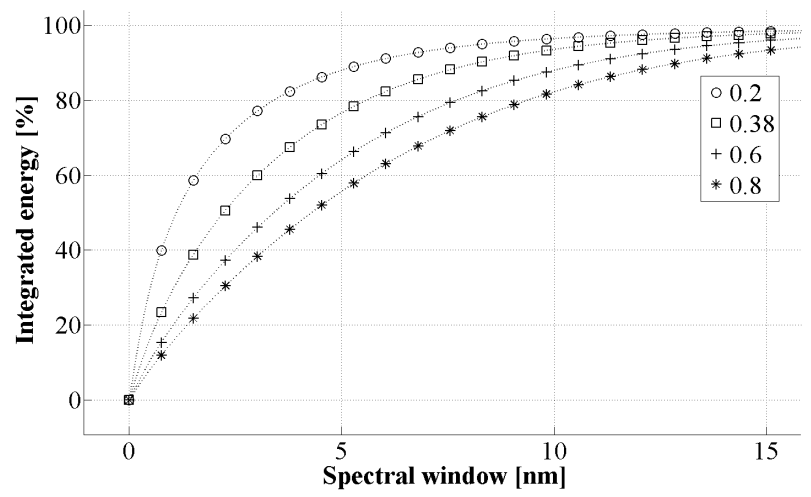

FIG. 3. Corresponding integrated DSP energy contained in the area centered at maximum spectrum power $\lambda_{S}=1064 \mathrm{~nm}$ for different values of fibre Kerr parameter $\gamma$ ranging from 0.2 to $0.8 \mathrm{~W}^{-1} \mathrm{~km}^{-1}$.

Interesting behaviors can be quantified by modifying the coherence time of input complex random process. In Fig. 4, as input PSD half-width is increased we have measured the output PSD enlargement $\eta=\Delta \nu_{O U T} / \Delta \nu_{I N}$ and the fraction of Raman energy considered as the total energy located at $\lambda>$ $1100 \mathrm{~nm}$. Both values increase in a monotonic way, however 
$\eta$ doesn't increase linearly at the difference with the Raman energy fraction. As we can see, Kerr nonlinearities and Raman induced frequency shift play an appreciable impact on coherence properties through amplification and propagation. However a close comparison between the modeling and experimental results show a spectrum spreading and Raman energy which is far more important in the experimental case [1]. We believe that adding Raman spontaneous emission in our model should solve this mismatch.

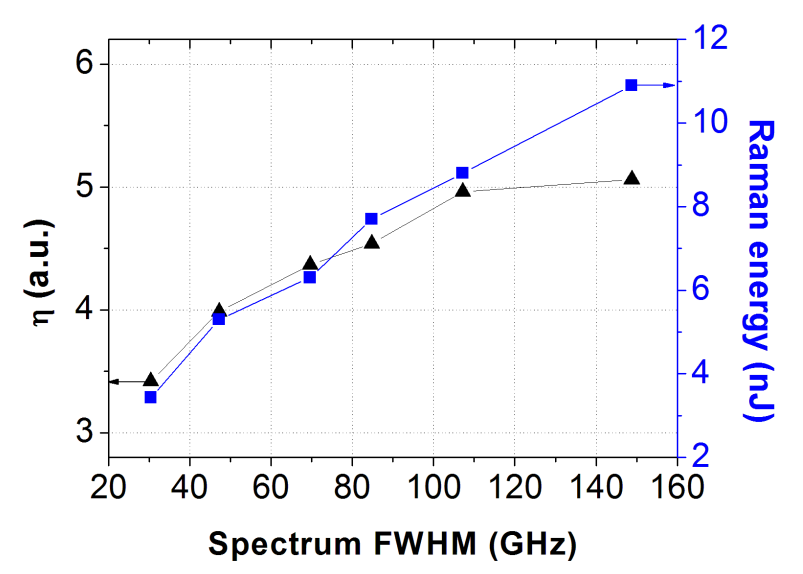

FIG. 4. Spectral spreading and corresponding integrated Raman energy at fibre output for a Lorentzian input spectrum shape with FWHM ranging from 30 to $150 \mathrm{GHz}$.

Finally, Fig. 5 shows the dependence of the PSD spreading with the choice of covariance function $C_{A\left[z_{n}\right]}$. PSD enlargement $\eta$ has been measured for increasing values of the laser diode pump power leading to a total linear amplification ranging from 11.9 to $18.6 \mathrm{~dB}$. As the pump power increases, in both cases $\eta$ is increasing too in a same way, however $\eta$ is two times bigger with an exponential covariance function (Lorentzian spectrum). Hence, accurate experimental measurement of $C_{A^{\left[z_{n}\right]}}$ is necessary in order to quantify spectrum enlargement at booster output.

\section{Conclusion}

We have presented in this paper a numerical simulation approach dedicated to the generation of partially coherent laser source and its propagation into a fibre amplifier. We have demonstrated an implementation of the RK4-IP method for solving the generalized nonlinear Schrödinger equation. The method exhibits a fourth order convergence and it is implemented together with an embedded Runge-Kutta method for adaptive step size control. Partially coherent laser source are modelled by means of complex Gaussian random processes. The choice of the circular embedding method for the generation of complex Gaussian random processes allows the simulation of partially coherent laser sources over a sufficiently large number of time sampling points (up to $2^{23}$ ) to ensure high accuracy and

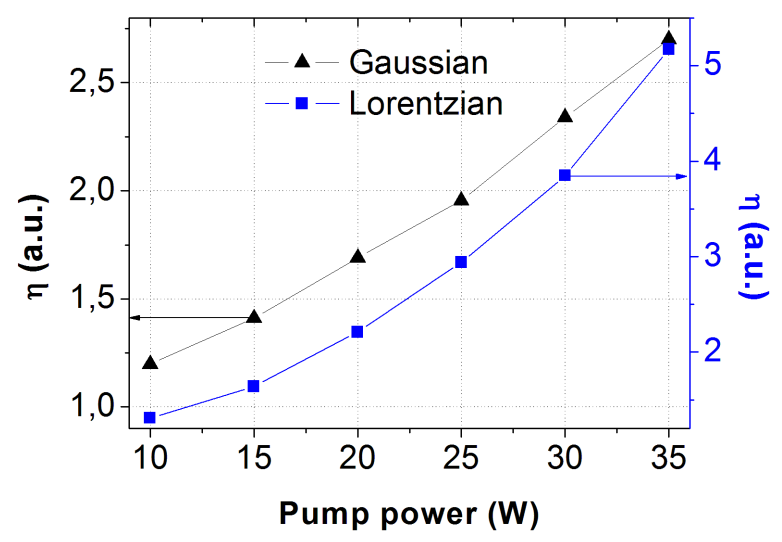

FIG. 5. Spectral spreading at fibre output for a respectively Gaussian and Lorentzian input spectrum shape with same FWHM of $130 \mathrm{GHz}$. The spectral spreading has been measured for an increasing pump power ranging from 10 to $35 \mathrm{~W}$.

wide span investigation. Besides computing speed has been optimized by the use of multi-threading technique for FFT computing and by parallel running of independent Gaussian random processes. Our numerical results show the ability of our numerical simulation software to bring physical statement to a pragmatic research topic such as high power nanosecond partially coherent pulse amplification in a $3 \mathrm{~m} \mathrm{Yb}^{3+}$ doped fibre. However, this numerical simulation software is for sure multipurpose and able to carry out studies in different photonic areas such as supercontinuum generation, or to deal with telecommunication concerns like data format transmission or optic functions based on four wave mixing.

\section{Acknowledgements}

The authors would like to warmly thank Pr. O. Boëffard and Dr. S. Le Maguer from IRISA (CNRS UMR 6074) Project team CORDIAL for their illuminating conversations on computer science, technical support and computing skills.

This work is supported by the Conseil Régional de Bretagne in the framework of the Green Laser project.

\section{Références}

1. P. Beaure D'Augères, A. Mugnier, D. Pureur, and T. Chartier. Time-resolved spectral analysis for nonlinear effects characterization in pulsed lasers. In Nonlinear Optics and Applications IV, pages 77280S-77280S-10, SPIE Photonics Europe, Bruxelles, Belgique, 2010.

2. G. Agrawal. Nonlinear Fiber Optics. Academic Press, 3rd edition, 2001.

3. K. Okamoto. Fundamentals of Optical Waveguides. Optics and Photonics. Elsevier, 2006.

4. D. Hollenbeck and C. D. Cantrell. Multiple-vibrational-mode model for fiber-optic Raman gain spectrum and response function. J. Opt. Soc. Am. B, 19(12) :2886-2892, 2002. 
5. R. H. Stolen, J. P. Gordon, W. J. Tomlinson, and H. A. Haus. Raman response function of silica-core fibers. J. Opt. Soc. Am. B, 6(6) :1159-1166, 1989.

6. O.V. Sinkin, R. Holzlöhner, J. Zweck, and C.R. Menyuk. Optimization of the Split-Step Fourier method in modeling optical-fiber communications systems. J. Lightwave Technol., 21(1) :61, 2003.

7. T.N. Nguyen, M. Gay, L. T. Bramerie, T. Chartier, and J.C. Simon. Noise reduction in 2R-regeneration technique utilizing selfphase modulation and filtering. Opt. Exp., 14(5) :1737-1747, 2006.

8. J. Hult. A fourth-order Runge-Kutta in the Interaction Picture method for simulating supercontinuum generation in optical fibers. J. Lightwave Technol., 25(12) :3770-3775, 2007.

9. S. Balac, A. Fernandez, F. Mahé, and R. Texier-Picard. The Interaction Picture method for solving the generalised nonlinear Schrödinger equation for wave propagation in optical fibres : theoretical and experimental comparison with split-step methods. CNRS FOTON technical report, http ://hal.archives-ouvertes.fr, 2012.

10. J.C. Butcher. Numerical Methods for Ordinary Differential Equations. John Wiley and Sons, 2008.

11. M. Frigo and S.G. Johnson. The design and implementation of FFTW3. Proceedings of the IEEE, 2(93):216-231, 2005.

12. A Heidt. Efficient adaptive step size method for the simulation of supercontinuum generation in optical fibers. J. Lightwave Technol., 27(18) :3984-3991, 2009.

13. S. Balac and A. Fernandez. Comparison of adaptive stepsize control strategies for solving the Generalised Non-Linear Schrödinger Equation in optics by the Interaction Picture method. CNRS FOTON technical report, http ://hal.archivesouvertes.fr/hal-00740771, 2012.

14. J. R. Dormand and P. J. Prince. New Runge-Kutta algorithms for numerical simulation in dynamical astronomy. Celestial Mech. $18: 223-232,1978$.

15. S. Balac and F. Mahé. Embedded Runge-Kutta scheme for stepsize control in the Interaction Picture method. Comput. Phys. Commun., (in press), 2013.

16. B. Gross and J. T. Manassah. Compression of the coherence time of incoherent signals to a few femtoseconds. Opt. Lett., 16(23) : 1835-1837, 1991.

17. C. R. Dietrich and G. N. Newsam. A fast and exact method for multidimensional gaussian stochastic simulations. Water Resour. Res., 29 :2861-2870, 1993.

18. A. T. A. Wood and G. Chan. Simulation of stationary Gaussian processes in $[0,1]^{d}$. J. Comput Graph. Stat., 3 :409-432, 1994.

19. G. Chan and A. T. A. Wood. Simulation of stationary Gaussian vector fields. Stat. Comput., 9(4) :265-268, 1999.

20. D. B. Percival. Exact simulation of complex-valued Gaussian stationary processes via circulant embedding. Signal Process., $86: 1470-1476,2006$.

21. J.W. Goodman. Statistical Optics. Wiley Classics Library, 2000. 